\title{
Falsely elevated salicylate levels
}

Dear Editor,

We report a case of falsely elevated salicylate levels resulting from hyperlipidemia in which improper sample processing led to reproducible errors in analysis. While hyperlipidemia has been shown to alter spectrophotometric laboratory results, spuriously elevated salicylate levels resulting from hyperlipidemia have not been previously reported in the literature.

A 38-year-old male with a history of nonischemic cardiomyopathy and hypothyroidism presented to an outside hospital (OSH) with altered mental status of worsening severity over approximately 1 week. The patent had fallen multiple times during the day of presentation and was noted to be confused by family. He admitted to drinking a small amount of an ethanolcontaining beverage that day with dinner. The patient had experienced multiple episodes of syncope during the previous 2 weeks that appeared to be brought on by exertion. He also claimed to have worsening dyspnea. The patient stated he had a history of depression but denied current suicidal intent or recent excess drug use, including aspirin. Past medical history was significant for cardiomyopathy associated with myocarditis, along with hyperlipidemia, hypothyroidism, and depression. His most recent echocardiogram revealed an ejection fraction of $45 \%$. The patient's medications included gabapentin, atorvastatin, buspirone, furosemide, magnesium oxide, lisinopril, digoxin, carvedilol, levothyroxine, and aspirin $162 \mathrm{mg}$ per day.

Initial vital signs were reported as follows: blood pressure $60 / 31$, pulse 108 , temperature 99.7 orally, respirations 20, oxygen saturation $96 \%$ on room air. Physical examination revealed an alert patient who was oriented to person, place, and time and showed no evidence of cognitive dysfunction, tachypnea, or hyperpnea. He had a few abrasions on his forehead, pupils were $2 \mathrm{~mm}$ - equal and reactive, skin was dry, and mucous membranes were moist. The patient had a regular heart rate and an otherwise unremarkable exam. Laboratory studies at the OSH revealed: sodium $114 \mathrm{mmol} / \mathrm{L}$ (ref. 135-145 $\mathrm{mmol} / \mathrm{L}$ ), potassium 2.8 $\mathrm{mmol} / \mathrm{L}$ (ref. 3.6-5.0 mmol/L), chloride $80 \mathrm{mmol} / \mathrm{L}$ (ref. 101-111 $\mathrm{mmol} / \mathrm{L}$ ), total $\mathrm{CO}_{2} 21 \mathrm{mmol} / \mathrm{L}$, and blood glucose $140 \mathrm{mg} / \mathrm{dL}$. Calculated anion gap was $13 \mathrm{mmol} / \mathrm{L}$. Complete blood count showed a hemoglobin of $12.7 \mathrm{~g} / \mathrm{dL}$ and a platelet count of $150,000 / \mu \mathrm{L}$. The patient's ethanol level was $34 \mathrm{mg} / \mathrm{dL}$, APAP 7.8 $\mu \mathrm{g} / \mathrm{mL}$, and salicylate $109 \mathrm{mg} / \mathrm{dL}$ (ref. $<3 \mathrm{mg} / \mathrm{dL}$ ). Digoxin level was reported as $0.5 \mathrm{ng} / \mathrm{mL}$ (ref. 1.0-2.0 ng/mL). Arterial blood gas analysis revealed: $\mathrm{pH} 7.48, \mathrm{pCO}_{2} 41 \mathrm{mmHg}$, and $\mathrm{pO}_{2} 61 \mathrm{mmHg}$. Noncontrast brain CT did not reveal any acute abnormality. His ECG showed sinus rhythm at 101 beats per minute, with multiple premature ventricular beats in a ventricular trigeminal pattern.
Based on the reported salicylate level, the regional poison center was contacted and the on-call medical toxicologist was consulted. Alkalinization therapy was recommended and transfer to a tertiary care center with dialysis capability was arranged. Secondary to incongruities between the patient's overall appearance and laboratory values, a repeat collection salicylate level was obtained shortly after the first collection, which was reported to be $77 \mathrm{mg} / \mathrm{dL}$.

Upon arrival at the tertiary care center the patient was evaluated by the toxicology service. He was alert, oriented, and normotensive, with a normal heart rate and without tachypnea or hyperpnea. Upon further questioning, the patient continued to deny intentional or accidental overdose of his medications, including aspirin. Initial labs at the tertiary center revealed: sodium $132 \mathrm{mmol} / \mathrm{L}$, potassium $3.1 \mathrm{mmol} / \mathrm{L}$ (ref. 3.5-4.5 mmol/L), chloride $94 \mathrm{mmol} / \mathrm{L}$, and total $\mathrm{CO}_{2} 20 \mathrm{mmol} / \mathrm{L}$, with an anion gap of $18 \mathrm{mmol} / \mathrm{L}$. AST and ALT were elevated at $328 \mathrm{U} / \mathrm{L}$ and $109 \mathrm{U} / \mathrm{L}$, respectively. His serum APAP concentration was 4.8 $\mu \mathrm{g} / \mathrm{mL}$ and his salicylate level was below the laboratory reporting limit ( $<5 \mathrm{mg} / \mathrm{dL}$ ). The patient's total cholesterol and triglyceride levels were markedly elevated at $1133 \mathrm{mg} / \mathrm{dL}$ (ref. $<200 \mathrm{mg} / \mathrm{dL}$ ) and $6390 \mathrm{mg} / \mathrm{dL}$ (ref. 50-320 mg/dL), respectively. The patient was treated supportively, his transaminases improved, and he was discharged with a diagnosis of orthostatic hypotension, hyperlipidemia, and hepatic steatosis.

The majority of clinical salicylate assays utilize spectrophotometry as a means of detecting and quantifying serum salicylate concentrations. The standard technique of salicylate detection and quantification utilizes an enzymatic method (salicylate hydroxylase) to alter the absorption of spectrophotometric light in the presence of salicylate [1]. Both the OSH and the tertiary care center employed this spectrophotometric method for salicylate quantification. In the presence of lipemia, it has become standard practice in many laboratories to use a polymer-based lipemia-clearing reagent, such as LipoClear, or ultracentrifugation to alleviate interference by high concentrations of serum lipids as a result of their light-absorptive properties. This is done not only for salicylate testing, but also for a variety of other analytes. However, in this patient, the OSH did not use this lipemia-clearing reagent or ultracentrifugation prior to sample analysis. This issue was brought to the attention of the OSH laboratory personnel, who graciously discussed and reviewed their analysis with us. Per the manufacturer's instructions, the OSH laboratory was aware that lipemic specimens should be delipidated by reagent or ultrafiltration, but they were unable to provide this service and were unaware of how lipemia would affect results. The laboratory of the OSH also reran the original sample (without the lipemia-clearing 
reagent) multiple times with results in $90 \mathrm{mg} / \mathrm{dL}$ range each time. The original sample was then sent from that OSH to a reference laboratory, where the delipidation step was performed, with a resultant nonquantifiable salicylate level $(<2.0 \mathrm{mg} / \mathrm{dL})$.

On review of the literature, there is no previously published report of lipemia-related spurious elevation in serum salicylate levels. However, hyperlipidemia is known to result in altered drug levels of other xenobiotics as well as endogenous compounds [2]. In addition, documentation provided by the manufacture of the analytical instrument used by the OSH suggests that salicylate levels might similarly be affected by hyperlipidemia. It is well known that hyperlipidemia may result in pseudohyponatremia [3]. In retrospect, the serum sodium level of $114 \mathrm{mmol} / \mathrm{L}$ reported by the OSH could have prompted earlier recognition of the possibility of laboratory error, further leading to the recognition that salicylate toxicity was not present in the patient, and possibly preventing unnecessary transfer.

We present this case to illustrate how lipemic samples may falsely elevate salicylate levels when the procedure of delipidation is not applied, and to help prevent unnecessary further testing or hospital transfer when false positive tests are obtained.
Nathan P. Charlton, MD

David T. Lawrence, DO

Kevin L. Wallace, MD

Division of Medical Toxicology

Blue Ridge Poison Center

University of Virginia

1222 Jefferson Park Avenue, Suite 4611

Charlottesville, VA 22903

NPC8A@hscmail.mcc.virginia.edu

\section{REFERENCES}

1. You K, Bittikofer JA. Quantification of salicylate in serum by use of salicylate hydroxylase. Clin Chem 1984;30(9): 1549-1551.

2. Baer DM, Paulson RA. The effect of hyperlipidemia on therapeutic drug assays. Ther Drug Monit 1987;9(1):72-77.

3. Goh KP. Management of hyponatremia. Am Fam Physician 2004;69(10):2387. 